\title{
Susceptibility-Weighted MR Imaging Hypointense Rim in Progressive Multifocal Leukoencephalopathy: The End Point of Neuroinflammation and a Potential Outcome Predictor
}

\author{
(D) M.M Thurnher, (D). Boban, (D)A. Rieger, and ${ }^{(D)}$ E. Gelpi
}

\begin{abstract}
BACKGROUND AND PURPOSE: Progressive multifocal leukoencephalopathy (PML) represents a life-threatening demyelinating disorder of the brain caused by reactivation of a rare opportunistic infection with JC Polyomavirus. The aims of this study were to describe the incidence of a susceptibility-weighted imaging hypointense rim in patients with multifocal leukoencephalopathy and to explore the histologic correlates and prognostic value of the rim with regard to the clinical outcome.
\end{abstract}

MATERIALS AND METHODS: This retrospective study included 18 patients with a definite diagnosis of progressive multifocal leukoencephalopathy. Ten patients were HIV-positive, 3 patients had natalizumab-associated progressive multifocal leukoencephalopathy, 1 patient had multiple myeloma, 3 patients had a history of lymphoma, and 1 was diagnosed with acute myeloid leukemia. Patients were divided into short- (up to 12 months) and long-term (>12 months) survivors. A total of 93 initial and follow-up MR imaging examinations were reviewed. On SWI, the presence and development of a hypointense rim at the periphery of the progressive multifocal leukoencephalopathy lesions were noted. $\mathrm{A}$ postmortem histologic examination was performed in 2 patients: A rim formed in one, and in one, there was no rim.

RESULTS: A total of 73 progressive multifocal leukoencephalopathy lesions were observed. In 13 (72.2\%) patients, a well-defined thin, linear, hypointense rim at the periphery of the lesion toward the cortical side was present, while in $5(27.8 \%)$ patients, it was completely absent. All 11 long-term survivors and 2 short-term survivors presented with a prominent SWI-hypointense rim, while 5/7 short-term survivors did not have this rim.

CONCLUSIONS: The thin, uniformly linear, gyriform SWI-hypointense rim in the paralesional U-fibers in patients with definite progressive multifocal leukoencephalopathy might represent an end-point stage of the neuroinflammatory process in long-term survivors.

ABBREVIATIONS: $J C V=J C$ Polyomavirus; PML = progressive multifocal leukoencephalopathy

$\mathbf{P}$ rogressive multifocal leukoencephalopathy (PML) is an infectious demyelinating disease of the central nervous system, caused by reactivation and replication of the JC Polyomavirus $(J C V) .{ }^{1}$ Although PML was traditionally associated with HIV/ AIDS, a shift toward patients on immunomodulation therapy has been observed in the past decade. ${ }^{2}$ Furthermore, PML has also

Received March 5, 2019; accepted after revision April 9.

From the Departments of Biomedical Imaging and Image-Guided Therapy (M.M.T., J.B.) and Dermatology (A.R.) and Institute of Neurology (E.G.), University Hospital Vienna, Medical University of Vienna, Vienna, Austria; and Neurological Tissue Bank of the Biobanc-Hospital Clinic-Institut dlnvestigacions Biomediques August Pi i Sunyer (E.G.), Barcelona, Spain. Dr Boban's permanent address is University of Novi Sad, Faculty of Medicine, Novi Sad, Serbia.

Please address correspondence to Majda M. Thurnher, MD, University Hospital Vienna, Department of Biomedical Imaging and Image-Guided Therapy, Medical University of Vienna, Waehringer Guertel 18-20, A-1090 Vienna, Austria; e-mail: majda.thurnher@meduniwien.ac.at

Indicates article with supplemental on-line table.

Indicates article with supplemental on-line photo.

http://dx.doi.org/10.3174/ajnr.A6072 been reported in patients with immune deficits associated with hematologic disorders. ${ }^{3,4}$ The MR imaging features of PML include single or multiple T2-weighted and FLAIR-hyperintense white matter lesions. ${ }^{5,6}$ Lesion borders are usually sharply demarcated toward the gray matter and blurred toward the deep white matter or ventricles, with absent or only faint contrast enhancement. ${ }^{7}$ On diffusion-weighted imaging, a high-signal-intensity rim of restricted diffusion that represents active demyelination has been described. ${ }^{8}$ To date, no specific features on conventional MR images have been identified as predictive of patient survival. ${ }^{9}$

Recently, the presence of a dark rim adjacent to the cortex or in the deep cortical layers on susceptibility-weighted imaging was reported in 2 patients with PML. ${ }^{10}$ Data based on quantitative susceptibility mapping of the lesions suggested a paramagnetic (blood product or nonheme iron deposits) effect. ${ }^{11}$ However, the mechanism that led to the susceptibility change remains unclear, because no histologic correlation was performed. SWI is a recently introduced MR imaging technique that has been used to 
evaluate hemorrhage, quantify brain iron content, and identify brain calcifications. $^{12}$

The aims of this retrospective study were to describe the incidence of an SWI-hypointense rim in patients with PML, to elucidate the histologic correlate of this rim, and to explore the prognostic value of the observed SWI features with regard to the clinical outcome.

\section{MATERIALS AND METHODS \\ Study Population}

A total of 18 consecutive patients with a definite diagnosis of PML ( 13 male and 5 female patients), with an average age of 50.56 years (range, 26-75 years) who underwent MR imaging of the brain at our institution from September 2009 to December 2018 were included in this institutional review board (Ethics Committee of the Medical University Vienna)-approved, retrospective study. Patients with no SWI in any of the MR imaging examinations were excluded. Ten (55.6\%) patients were HIV-positive, 3 (16.7\%) had natalizumab-associated PML, 1 patient (5.6\%) had multiple myeloma, 3 patients $(16.7 \%)$ had lymphoma, and 1 patient $(5.6 \%)$ had acute myeloid leukemia and bone marrow transplantation. Of the 3 patients with lymphoma, 1 patient had large-cell lymphoma, 1 patient had small-cell B-cell lymphoma in remission, 1 patient was being treated with rituximab, and 1 patient had nonHodgkin lymphoma. In 1 patient, herpes simplex virus type 2 encephalitis was also diagnosed, and 5 patients had a chronic coinfection with the hepatitis $\mathrm{C}$ virus. The diagnosis of definite PML in all patients was established on the basis of clinical data, imaging findings, and detection of DNA copies of the JCV in the CSF using the polymerase chain reaction, according to the American Academy of Neurology criteria (American Academy of Neurology guidelines). ${ }^{13}$

For HIV-positive patients, we included the following clinical data: current and nadir CD4 count, plasma viral load, duration of the HIV infection; neurologic signs and symptoms at the PML onset; and duration of antiretroviral therapy. In 2 HIV-negative patients, the neurologic signs and symptoms and the time range between the onset of PML and the underlying disease were noted. For natalizumab-associated PML, we included the following clinical data: the year of the diagnosis of multiple sclerosis and the duration of natalizumab therapy.

Patients were classified as long- or short-term survivors, with a survival time of up to 12 months for short-term survivors and longer than 12 months for long-term survivors.

\section{MR Imaging Protocol}

Ninety-one MR imaging examinations were performed in 18 patients on different clinical MR imaging scanners (Avanto, Siemens, Erlangen, Germany, 1.5T; Tim Trio, Siemens, 3T; Ingenia, Philips Healthcare, Best, the Netherlands, 1.5T; Achieva, Philips Healthcare, 3T). The MR imaging protocol included the following sequences: axial FLAIR, coronal T2-weighted, axial T1weighted, and axial diffusion-weighted MR imaging in all patients. Postcontrast T1WI was performed in all patients $(82 / 91$ examinations). SWI was performed in all patients (74/91 examinations). Susceptibility-weighted imaging was performed with the following parameters: FOV $=230 \times 190 \times 130 \mathrm{~mm}$ (ante- rior-posterior $\times$ right-left $\times$ height-feet), voxel size $=0.6 \times$ $0.6 \times 1 \mathrm{~mm}$, reconstruction matrix $=960, \mathrm{TE} / \mathrm{TR}=7.12 / 31 \mathrm{~ms}$, flip angle $=17^{\circ}$ with no water or fat suppression used $(3 \mathrm{~T} \mathrm{MR}$ imaging unit, Tim Trio). Parameters for the SWI sequence on the 1.5T MR imaging unit (Avanto) were the following: $\mathrm{FOV}=$ $230 \times 186 \times 150 \mathrm{~mm}$, voxel size $=0.85 \times 1 \times 2 \mathrm{~mm}$, reconstruction matrix $=576, \mathrm{TE} / \mathrm{TR}=12 / 35 \mathrm{~ms}$, flip angle $=20^{\circ}$ with no water or fat suppression.

\section{Image Analysis}

Overall, $91 \mathrm{MR}$ imaging examinations were analyzed, with a mean of 4.17 follow-up imaging studies per patient (range, 0-11 studies).

All imaging studies were analyzed independently on a digital workstation by 2 readers and in a final consensus read (M.M.T., J.B.). The morphologic findings of lesions were described, including the lesion location, the size and number, and lesion features on pre- (T2WI, FLAIR, T1WI, DWI) and postcontrast T1WI. On SWI, the presence or absence of a hypointense rim at the periphery of the PML lesions was noted. The development of this rim with time was estimated, compared with prior images, and expressed in weeks. In addition, the correlation of the onset of an SWI-hypointense rim, DWI abnormalities, and the presence of contrast enhancement was explored.

\section{Neuropathology}

A postmortem neuropathologic examination was performed in 2 patients (patients 2 and 16 in the On-line Table). Formalin-fixed and paraffin-embedded tissue blocks from multiple brain areas, including a whole, representative hemispheric section, were processed for conventional histologic and immunohistochemical stains. Five- $\mu \mathrm{m}$-thick sections were stained with hematoxylineosin, as well as Klüver-Barrera (Luxol fast blue and Nuclear fast red) for the labeling of myelin sheaths, and, in selected areas, with Prussian blue for the detection of iron. Immunohistochemistry was applied for the detection of the JCV and for the evaluation of astroglia (glial fibrillary acidic protein, GFAP; Dako, Glostrup, Denmark) and microglia (HLA-DR; Dako).

\section{Statistical Analysis}

Descriptive statistics were used for various findings on MR imaging. For categoric data, we used frequencies and percentages, while for continuous data, range and median values were used. Survival was estimated using Kaplan-Meier estimates. The results are presented in a pictorial-review format.

\section{RESULTS}

\section{Clinical Data}

All patients fulfilled the American Academy of Neurology criteria for definite PML. Among HIV-positive subjects, the median duration of HIV infection before the onset of PML was 5.88 years (range, 0-23 years), the mean nadir CD4+ was 89 cells $/ \mathrm{mL}$ (range, 15-199 cells/mL), the mean current CD4+ T-cell count (at the onset of PML) was 120.4 cells/mL (range, 15-292 cells $/ \mathrm{mL}$ ), and the mean viral load at the time of PML diagnosis and before combination antiretroviral therapy (cART) initiation was 3.83 $\mathrm{IU} / \mathrm{mL}$ (range, 2.59-6.04 IU/mL) and it was $1.29 \mathrm{IU} / \mathrm{mL}$ (below the 
Survival Functions

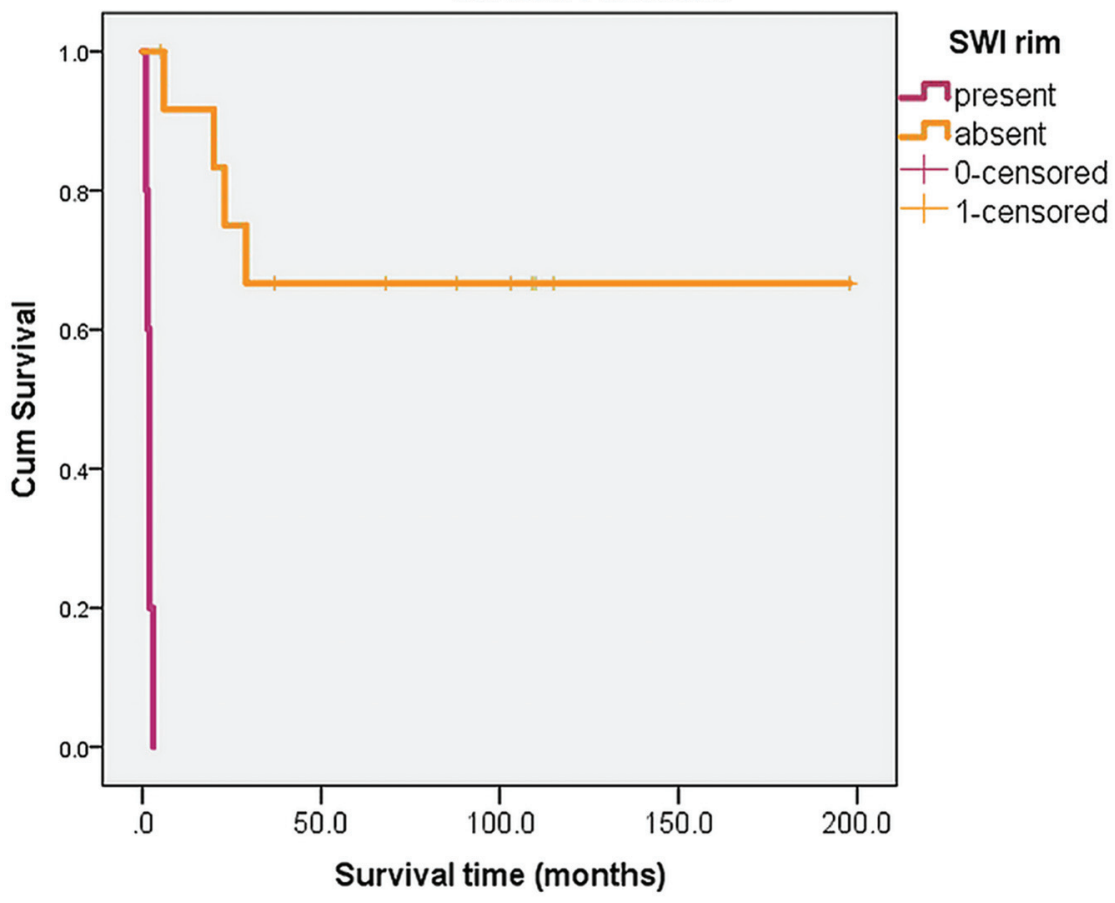

FIG 1. Kaplan-Meier survival analysis, with differences in survival and outcome between patients with (in purple) and without (in orange) an SWI-hypointense rim.

detection level) after cART initiation. In 4 (40\%) patients, PML was the initial manifestation of the HIV infection/AIDS.

Neurologic symptoms at presentation are listed in the On-line Table.

Three patients $(18.75 \%)$ were receiving HAART before the PML diagnosis (median duration of cART was 5.33 years; range, 3-9 years). In all patients, DNA copies of the JCV were detected in the CSF. One patient presented with B-cell chronic lymphocytic leukemia. In 1 patient with acute myeloid leukemia and bone marrow transplantation, the diagnosis of PML was made 3 months after the bone marrow transplantation. One patient was under immunomodulatory therapy for multiple myeloma. Of the 3 patients with natalizumab PML, all had relapsing-remitting MS diagnosed 5-12 years (mean, 8.3 years) before the onset of PML. The duration of natalizumab therapy was 3.6 years ( 4 years, 3 years, 3 years, respectively).

Seven patients were classified as short-term survivors, with a mean survival time of 2.93 months (range, 1-6 months). The cause of death was PML in 5 patients, and 1 patient died due to a non-PML cause (lung embolism). One short-term survivor (with a defined SWI-hypointense rim) has survived 5 months and is alive and stable at the moment. In 11 patients classified as longterm survivors, the mean survival time was 81.82 months (range, 20-198 months).

The results of the survival analysis clearly demonstrate differences in survival and outcome between patients with (in purple) and without (in orange) an SWI-hypointense rim (Fig 1).

\section{MR Imaging Findings}

A total of 73 PML lesions were observed in 18 patients, involving the frontal $(31 / 42.4 \%)$, parietal $(9 / 12.3 \%)$, temporal $(5 / 6.8 \%)$, and occipital (6/8.2\%) lobes, and the insular region $(4 / 5.5 \%)$, the external capsule $(1 / 1.4 \%)$, the internal capsule $(2 /$ $2.8 \%)$, the thalamus $(1 / 1.4 \%)$, the corpus callosum (1/1.4\%), the pons ( $1 /$ $1.4 \%)$, and the cerebellum (9/12.3\%). All lesions had typical MR imaging features-that is, high signal on T2WI/ FLAIR and marked low signal on T1WI. Only 2 of 18 patients (11.1\%) showed a peripheral rim of restricted diffusion on DWI with corresponding low ADC values.

Contrast enhancement was present in $10 / 18(55.6 \%)$ patients. According to the enhancement pattern, patients were divided into 4 groups: 1) "No enhancement" was seen in 8/18 (44.4\%) patients (2 patients with lymphoma, 2 with MS, 1 patient with multiple myeloma, and 3 HIV-positive patients); 2) "faint enhancement" was detected in 5/18 (27.8\%) patients (3 HIV-positive patients, 1 patient with MS, and 1 patient with lymphoma); 3) an "inflammatory pattern" was seen in $2 / 18$ (11.2\%) patients (1 HIV-positive patient, 1 patient with acute myeloid leukemia and bone marrow transplantation); and 4) an "immune reconstitution inflammatory syndrome pattern" was present in 1 of $18(5.6 \%)$ patients (an HIV-positive patient).

\section{SWI Findings}

SWI findings were available for all 18 patients. On SWI, a welldefined thin, linear, hypointense rim at the periphery of the lesion, located toward the cortical side (Fig 2), was present in 13/18 patients $(72.2 \%)$, while in 5 patients $(27.8 \%)$, the rim was completely absent (Fig 3). Of 16 patients in whom SWI was performed within the first 2 months after the PML diagnosis, the presence of an SWI-hypointense rim was detected in 11 patients, with the mean time to formation of 2.8 weeks (range, 1-8 weeks). In 1 patient, the first signs of an SWI-hypointense rim were observed 13 weeks after the initial MR imaging examination, but the follow-up examination was not performed within the first 2 months. In addition, in 1 patient, the first SWI data were available 6 years after the diagnosis, with a positive SWI-hypointense rim (burnout lesion). The SWI-hypointense rim gradually increased in thickness with time, spreading continuously along the juxtacortical white matter following the gyral architecture.

\section{Correlation of SWI and Contrast Enhancement and DWI Findings}

At the time of the first appearance of the SWI dark rim, only 4 patients had contrast enhancement. However, this contrast enhancement was not located at the SWI-hypointense rim, but either on the peripheral rim of the PML lesion toward the ventricle or closer to the center of the PML lesion (faint or dotty enhancement). Contrast enhancement was not observed in the region of 
the SWI-hypointense rim in any of the examinations in which postcontrast T1WI was available for analysis.

In 2 patients (patients 8 and 12) in whom a DWI-hyperintense rim was present, SWI did not show a dark rim. In 1 patient (patient 12) in whom follow-up was available, 3 weeks after the initial examination, the DWI rim disappeared and the SWI rim was detected (Fig 4).

\section{Neuropathologic Findings}

A neuropathologic examination was performed in 2 patients, (patients 2 and 16 in the On-line Table); in 1 patient, an SWI rim was

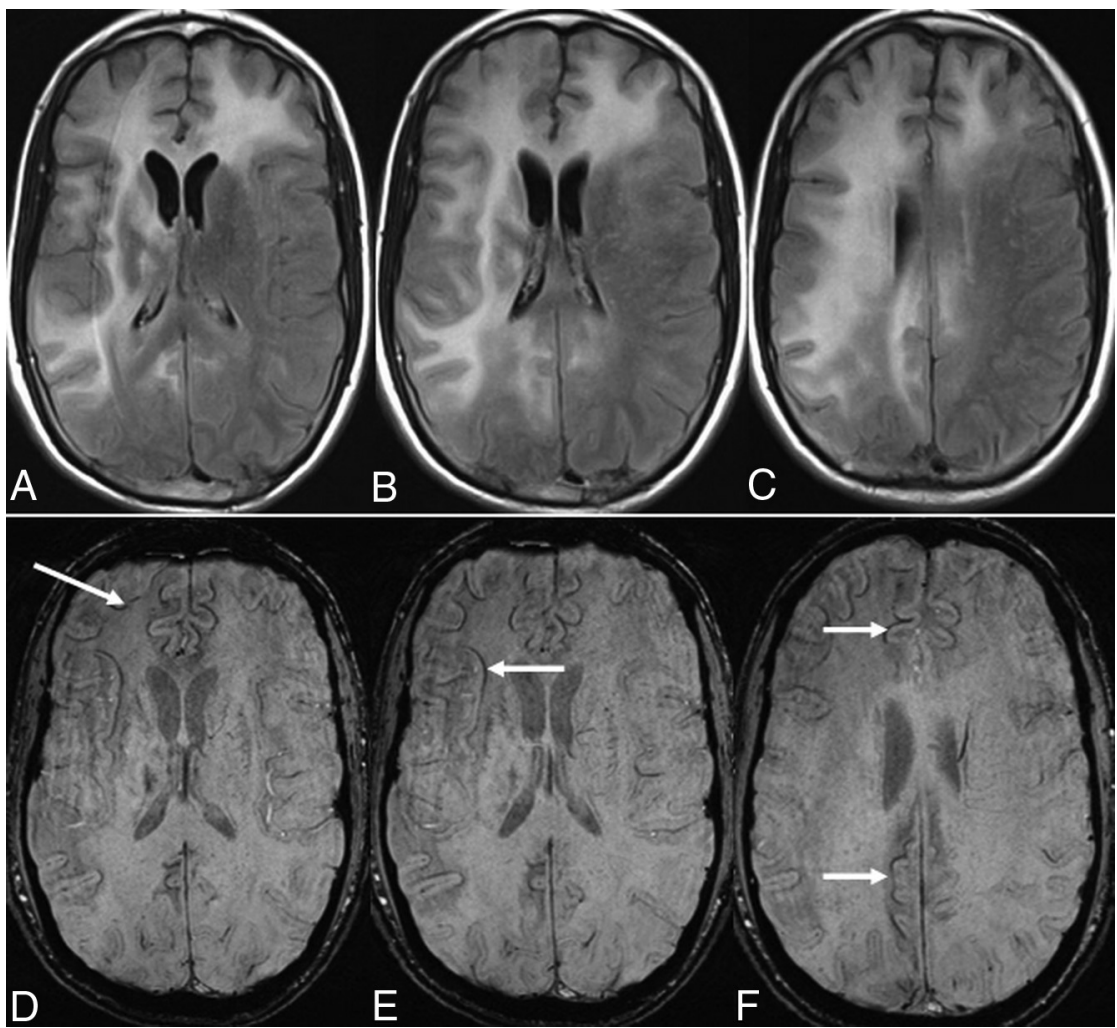

FIG 2. On axial FLAIR images $(A-C)$, multifocal PML lesions are observed in both frontal lobes, the capsula interna and externa, and the right parietal lobe. A linear, relatively thin hypointense rim is observed on the cortical side of the lesions on axial SWI $(D-F)$ in a long-term survivor (9 years, still alive). present (patient 2), but no SWI rim was detected in the second patient (patient 16). Patient 2, with a survival time of 6 months, died of multiorgan dysfunction syndrome, and in patient 16 , the cause of death was PML.

In patient 2 (On-line Figure A1), gross examination showed multiple white matter lesions in both hemispheres, some of which were softened and yellowish and were most pronounced at the frontal regions with an already necrotic aspect. Histology showed features of an extensive inflammatory demyelinating pathology with prominent loosening of white matter, loss of myelin sheaths with relatively preserved axons, abundant macrophages, and perivascular lymphocytic infiltrates. No parenchymal bleeding was observed.

In patient 16 (On-line Figure B1), gross examination showed multiple and partly confluent and softened white matter lesions mainly in the left-brain hemisphere, extending from the frontal to the parietotemporal regions, with relative preservation of the overlying cortex. Histology confirmed the inflammatory and demyelinating nature of the lesion (On-line Figure B5). No parenchymal bleeding was detected.

In patient 2 with the faint SWI rim, the number of PML-infected cells was much lower than in the patient without an SWI rim (On-line Figure A5 and B5). In addition, there was much more prominent astrogliosis and microglial activation in the deep cortical layers and at the cortical-subcortical junction, including the U-fibers (On-line Figure A6 and A7). This feature was observed not only in areas overlying PML lesions but also in areas without focal lesions, but with diffuse white matter pallor and moderate gliosis. In both cases, a Prussian blue stain was performed for the detection of iron deposits. In patient 2 ,

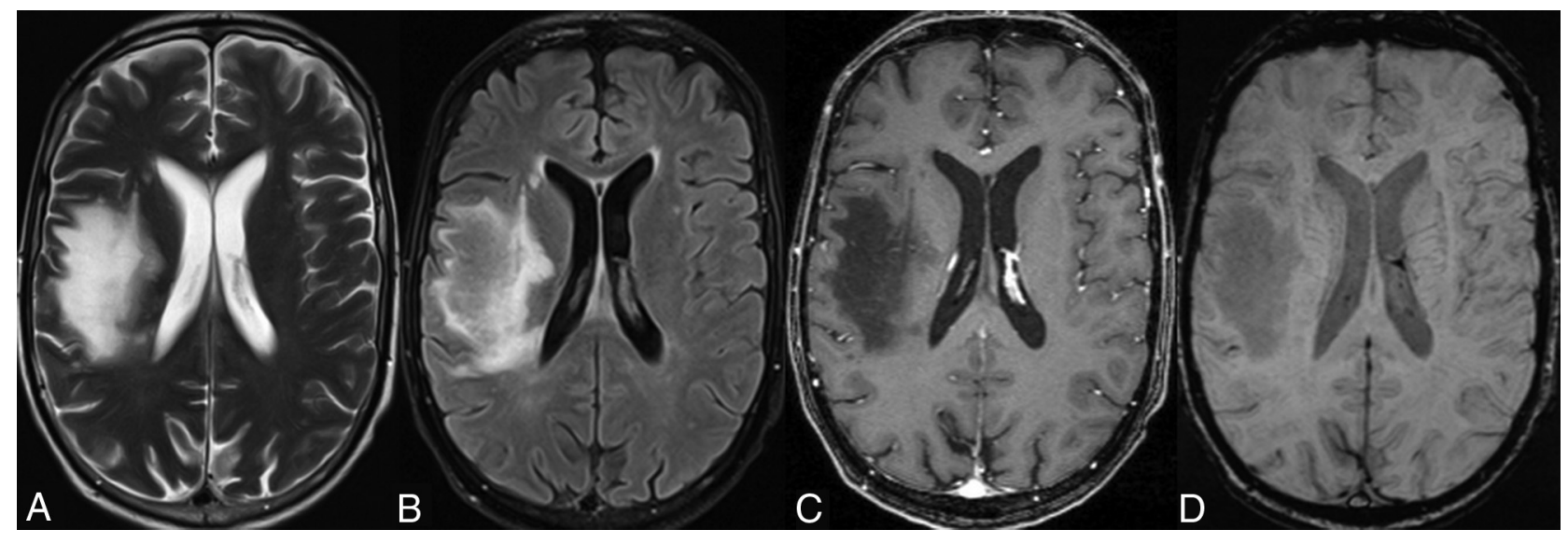

FIG 3. A typical T2-weighted $(A)$ and FLAIR $(B)$ hyperintense PML lesion is observed in the frontoparietal corona radiata on the right side. On the postcontrast T1-weighted axial image $(C)$, only a faint enhancement pattern is observed. On the axial SWI (D), there are no signs of a gyriform hypointense rim. The patient was classified as a short-term survivor (11 weeks). 

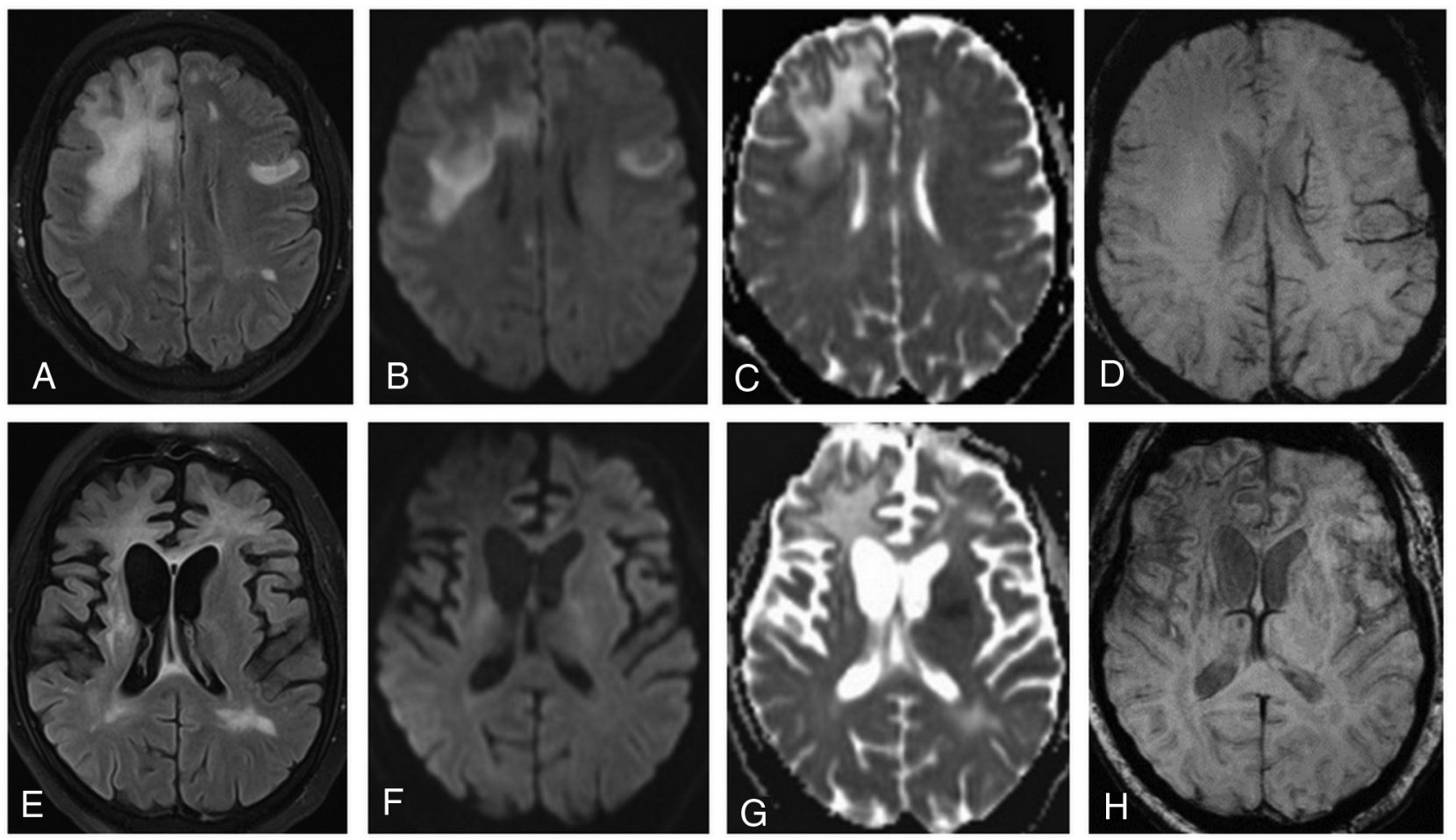

FIG 4. On an axial FLAIR image (A), bilateral high-signal-intensity lesions are detected. DWI clearly demonstrates a high-signal-intensity rim at the periphery of the lesion in the right frontal lobe on trace DWI $(B)$, with low ADC values $(C)$ indicating restricted diffusion. On SWI $(D)$, no abnormalities are seen. On follow-up MR imaging (3 months later), atrophic changes with dilation of the ventricles are observed in both hemispheres (more prominent in the right) (E). On DWI/ADC ( $F$ and $G)$, no hyperintense rim is present. On SWI $(H)$, a dark rim is clearly visible.

abundant blue pigment was observed within actively demyelinating lesions (On-line Figure A3, triple asterisks). In the surrounding areas, diffuse homogeneous cytoplasmic staining of pericapillary cells was also observed (On-line Figure A3, double asterisks). In contrast, around vessels with moderate chronic gliosis, there was glial pigment and non-Prussian blue pigment alternating with Prussian blue pigment (On-line Figure A3, asterisk). In patient 16, fewer perivascular Prussian bluepositive areas were detected.

\section{DISCUSSION}

PML represents a life-threatening demyelinating disease of the brain as a result of infection with the $J C V .{ }^{14}$ Since the AIDS epidemic, PML was reported exclusively in association with HIV, but more recently, there has been a shift toward other immunosuppressive conditions, including patients on immune-suppression therapy and patients with long-standing autoimmune inflammatory or hematologic malignancies. ${ }^{15,16}$ In addition, several case reports on immunocompetent patients developing PML have also been published. ${ }^{17,18}$

A relatively thin, uniformly linear SWI-hypointense rim was observed in 13 of 18 patients with PML in our study. The rim exclusively affected the U-fibers, following the cortical architecture, and did not correlate with other MR imaging findings. No signs of susceptibility changes were detected in the center of the PML lesions or on the side toward the deep white matter and the ventricle. In addition, infratentorial PML lesions showed no SWIhypointense rim formation. Eleven of 13 patients in whom the SWI-hypointense rim was present were long-term survivors, whereas in 5 of 7 short-term survivors, there was no SWI-hypointense rim detected. One of our short-term survivors died due to a non-PML cause, and the SWI-hypointense rim in this particular patient could not be seen in correlation with the short survival (patient 2 in the On-line Table). One short-term survivor is still alive (patient 17 in the On-line Table), and because the cutoff point of 12 months has not yet been reached, this patient was classified as a short-term survivor, though a positive clinical outcome is highly likely at the moment.

The detection of the linear hypointense rim in the region of the U-fibers on SWI sequences has only recently been described and was postulated to be a specific finding in PML lesions. ${ }^{10}$ Later, the same group reported the presence of such a rim in patients with chronic cerebral infarction and after encephalitis. ${ }^{19}$ The etiology of this rim was unresolved and was not fully explained. CarraDalliere et $\mathrm{al}^{11}$ suggested that an SWI-hypointense rim in patients with PML could be a consequence of paramagnetic material deposits, based on quantitative susceptibility mapping. In both studies, no histologic correlation of SWI findings was available.

Several morphologic phenomena occur at the edges of a PML lesion and beneath juxtacortical areas, mainly primary oligodendroglial dysfunction by JCV infection and consequent myelin degeneration, microglial activation, astroglial proliferation, and axonal impairment. Axonal damage can produce retrograde neuronal degeneration and lead to progressive cortical atrophy. It is known that PML also involves the U-fibers. Chronically activated glial cells and microglia/macrophages may contain high levels of iron and pigment within their cytoplasm. In addition, blood- 
brain barrier disruption caused by PML may lead to leakage of heme products into the perivascular space. Although diffuse homogeneous cytoplasmic staining of cells reflects the presence of ferritin, hemosiderin shows the typical granular particles in lysosomes. We found severe reactive changes at the corticosubcortical boundary surrounding the PML lesions, which were more prominent in the patient with a longer survival. We also observed a higher, but not exclusive, ferritin and hemosiderin content within the lesions in the patient with a longer survival than in the patient with a shorter survival, which was more abundant around small vessels and capillaries in the deep cortical layers and at the juxtacortical border.

A similar but less prominent SWI-hypointense rim was observed in the deep cortical layers of the precentral gyrus in patients with amyotrophic lateral sclerosis. On histopathologic studies, intracellular deposits of iron (in the form of ferritin) were confirmed in reactive microglial cells, located in the middle and deep layers of the motor cortex, in areas that were affected by neuronal loss and axonal degeneration. ${ }^{20} \mathrm{Kim}$ et $\mathrm{al}^{21}$ recently reported ferritin accumulation in proliferated microglial cells in severely demyelinated U-fibers in patients with adult-onset leukoencephalopathy, with axonal spheroids and pigmented glia, as well as treelike SWI hypointensities in the subcortical white matter. In recently published studies by van Duijn et $\mathrm{al}^{22}$ and Bulk et al, ${ }^{23}$ a bandlike $\mathrm{T} 2{ }^{*}$-hypointense cortical rim was observed on $7 \mathrm{~T}$ in a subset of patients with Alzheimer disease, which was believed to be derived from increased myelin-associated, nonheme iron in the middle layers of the cortex and Baillarger lines and was also found in disintegrated axons and microglial cells.

One possible explanation for the specific location of iron deposition in our patients with PML and in the above-mentioned disorders is the fact that subcortical U-fibers have a greater iron content than both cerebral gray and white matter, due to the high density of iron-rich oligodendrocytes, and are considered slow metabolic fibers with a slow myelin turnover. In addition, the region of $\mathrm{U}$-fibers has long medullary arterioles and venules in a gyriform distribution. ${ }^{24}$

The other important question to address is the cause of iron deposition in PML. In 1981, Konijn et $\mathrm{al}^{25}$ had already suggested that ferritin accumulation could be a nonspecific, acute-phase reactant in response to inflammation. It seems that accumulating iron in proliferated microglial cells is not a specific finding in any of the above-mentioned neurodegenerative diseases, but rather a pattern in progressive and long-standing neuroinflammation. The fact that siderin- and ferritin-laden macrophages were seen in the perivascular region, especially around the small capillaries at the gray-white matter interface, indicates the possibility of inflammation-induced blood-brain barrier disturbance, with extravasation of blood cells and subsequent perivascular deposition of iron and iron end products. If an SWI dark rim does, indeed, represent an effective chronic neuroinflammatory response to a brain injury, it should then be present in PML long-term survivors and could be considered a sign of end-stage inflammation. The absence of an SWI dark rim in our short-term survivors favors this hypothesis.

In a recently published study, a T1WI-hyperintense cortical signal on MR imaging has been reported in $61.2 \%$ of patients with
PML. ${ }^{26}$ A hyperintense cortical signal was detected adjacent to subcortical PML lesions on precontrast T1-weighted MR images and resembled the SWI-hypointense rim seen in our patients. In addition, atrophy of the cortical area affected was reported in $96.7 \%$ of cases with hyperintense cortical signal. SWI was available in 15 of 49 patients in that particular study, and none of them had any SWI abnormality. On histology, hyperintense cortical signal areas were associated with striking JCV-associated demyelination of the cortical and subcortical U-fibers, heavy macrophage infiltration, and a pronounced reactive gliosis in the deep cortical layers. ${ }^{26}$ There were neither microhemorrhages nor iron deposits in the postmortem histologic sections of our 2 patients with PML in whom histologic analysis was obtained.

The characteristic MR imaging finding suggestive of inflammation is the presence of contrast enhancement in a given lesion. In general, in HIV-related PML, enhancement is observed in only up to $15 \%$. This is different for natalizumab-associated PML, in which enhancement will be present in $43 \%$ of cases. ${ }^{27}$ In a recently described form of PML, called "inflammatory PML," prominent enhancement along the perivascular spaces was observed. ${ }^{28}$ These lesions show perivascular cuffing and inflammatory plasma cell infiltrates. ${ }^{29}$ It was suggested that this PML form represents the fulminant, active, lytic infection of white matter cells caused directly by the $J C V$.

In our study, we could not establish a relationship between the presence of contrast enhancement and SWI-hypointense rim formation. In 2 of our short-term survivors, MR imaging findings were consistent with an inflammatory PML pattern, and neither of them developed an SWI-hypointense rim. Therefore, it seems likely that the SWI rim indicates a transitory BBB disturbance, rather than fulminant infection of oligodendrocytes, as seen in inflammatory PML.

The fact that, in 2 patients (patients 8 and 12 in the On-line Table), the presence of the peripheral rim of restricted diffusion was only present initially (as long as the SWI rim was not detected) and disappeared later with the formation of the SWI rim further confirms the hypothesis that the SWI rim represents the end-stage of neuroinflammation. Further prospective studies with a clearly defined time period for the follow-up MR imaging examination may deliver data that will indicate the time range needed for an SWI rim to develop in long-term survivors.

One limitation of our study is the relatively small number of patients, which does not allow an estimation of the real incidence of an SWI dark rim in patients with PML. Ideally, prospective data should be collected from several institutions. Another limitation is the rather limited histopathologic data of only 2 patients. However, the patient with a dark SWI rim died soon after the PML diagnosis from a non-PML-related disease; thus, the changes observed on histology most probably reflect findings in long-term survivors.

\section{CONCLUSIONS}

The formation of a thin, uniformly linear, gyriform SWI-hypointense rim in the region of the paralesional $U$-fibers in patients with PML seems to represent an end-point stage of the neuroinflammatory process in long-term survivors. Its morphologic correlate could be related to the chronic glio-inflammatory reaction, with 
increased iron content in microglia, oligodendroglia, and astroglia, and is attributable to BBB leakage along the perivascular spaces.

\section{ACKNOWLEDGMENTS}

We are grateful to Mrs Irene Ebner of the Institute of Neurology of the Medical University of Vienna for the excellent technical neuropathologic work-up.

Disclosures: Majda M. Thurnher-UNRELATED: Royalties: Amirsys. Armin RiegerUNRELATED: Payment for Lectures Including Service in Speakers Bureaus: MSD, Gilead Sciences, GlaxoSmithKline, Comments: presentations in several ad boards; Travel/Accommodations/Meeting Expenses Unrelated to Activities Listed: Gilead Sciences, MSD, GlaxoSmithKline, Comments: invitations to join several HIV/AIDS congresses. * *Money paid to the institution.

\section{REFERENCES}

1. Fournier A, Martin-Blondel G, Lechapt-Zalcman E, et al. Immune reconstitution inflammatory syndrome unmasking or worsening AIDS-related progressive multifocal leukoencephalopathy: a literature review. Front Immunol 2017;8:577 CrossRef Medline

2. Carson KR, Focosi D, Major EQ, et al. Monoclonal antibody-associated progressive multifocal leucoencephalopathy in patients with rituximab, natalizumab, and efalizumab: a review from the Research on Adverse Drug Events and Reports (RADAR) project. Lancet Oncol 2009;10:816-24 CrossRef Medline

3. Gocmen R, Acar NP, Cagdas D, et al. Progressive multifocal leukoencephalopathy in a patient with lymphoma and presumptive hyper IgE syndrome. J Neurovirol 2017;23:632-36 CrossRef Medline

4. Al-Tawfiq JA, Banda RW, Daabil RA, et al. Progressive multifocal leukoencephalopathy (PML) in a patient with lymphoma treated with rituximab: a case report and literature review. J Infect Public Health 2015;8:493-97 CrossRef Medline

5. Thurnher MM, Donovan Post MJ. Neuroimaging in the brain in HIV-1-infected patients. Neuroimaging Clin N Am 2008;18:93-117, viii CrossRef Medline

6. Tan CS, Koralnik IJ. Progressive multifocal leukoencephalopathy and other disorders caused by JC virus: clinical features and pathogenesis. Lancet Neurol 2010;9:425-37 CrossRef Medline

7. Thurnher MM, Post MJ, Rieger A, et al. Initial and follow-up MR imaging findings in AIDS-related progressive multifocal leukoencephalopathy treated with highly active antiretroviral therapy. AJNR Am J Neuroradiol 2001;22:977-84 Medline

8. Narvid J, Rehani B, Talbott JF. Diagnostic performance of brain MRI in immune reconstitution inflammatory syndrome. J Neuroimaging 2016;26:303-08 CrossRef Medline

9. Post MJ, Thurnher MM, Clifford DB, et al. CNS-immune reconstitution inflammatory syndrome in the setting of HIV infection, Part I: overview and discussion of progressive multifocal leukoencephalopathy-immune reconstitution inflammatory syndrome and cryptococcal-immune reconstitution inflammatory syndrome. AJNR Am J Neuroradiol 2013;34:1297-307 CrossRef Medline

10. Miyagawa $M$, Maeda $M$, Umino $M$, et al. Low signal intensity in U-fiber identified by susceptibility-weighted imaging in two cases of progressive multifocal leukoencephalopathy. J Neurol Sci 2014; 344:198-202 CrossRef Medline

11. Carra-Dalliere C, Menjot de Champfleur N, Ayrignac X, et al. Quantitative susceptibility mapping suggests a paramagnetic effect in PML. Neurology 2015;84:1501-02 CrossRef Medline

12. Liu C, Li W, Tong KA, et al. Susceptibility-weighted imaging and quantitative susceptibility mapping in the brain. J Magn Reson Imaging 2015;42:23-41 CrossRef Medline
13. Berger JR, Aksamit AJ, Clifford DB, et al. PML diagnostic criteria: consensus statement from the AAN Neuroinfectious Disease Section. Neurology 2013;80:1430-38 CrossRef Medline

14. Pontillo G, Cocozza S, Lanzillo R, et al. Brain susceptibility changes in a patient with natalizumab-related progressive multifocal leukoencephalopathy: a longitudinal quantitative susceptibility mapping and relaxometry study. Front Neurol 2017;8: 294 CrossRef Medline

15. Davis MJ, Khan A, Royal W 3rd. Progressive multifocal leukoencephalopathy as the first manifestation of occult sarcoidosis: report and review of the literature. Neurologist 2013;19:26-29 CrossRef Medline

16. Sholten P, Krait P, Jacobs B. Posterior fossa progressive multifocal leukoencephalopathy: first presentation of an unknown autoimmune disease. BMJ Case Rep 2017;2017 CrossRef Medline

17. Grewal J, Dalal P, Bowman M, et al. Progressive multifocal leukoencephalopathy in a patient without apparent immunosuppression. J Neurovirol 2016;22:686-87 CrossRef Medline

18. Van de Kolk NM, Arts P, van Uden IW, et al. Progressive multifocal leukoencephalopathy in an immunocompetent patient. Ann Clin Transl Neurol 2016;3:226-32 CrossRef Medline

19. Umino M, Maeda M, Ii Y, et al. Low-signal-intensity rim on susceptibility-weighted imaging is not a specific finding to progressive multifocal leukoencephalopathy. J Neurol Sci 2016;362:155-59 CrossRef Medline

20. Kwan JY, Jeong SY, Van Gelderen P, et al. Iron accumulation in deep cortical layers accounts for MRI signal abnormalities in ALS: correlating 7 Tesla MRI and pathology. PLoS One 2012;7:e35241 CrossRef Medline

21. Kim M, Lee H, Cho HJ, et al. Pathologic correlation of paramagnetic white matter lesions in adult-onset leukoencephalopathy with axonal spheroids and pigmented glia. J Neuropathol Exp Neurol 2017; 76:924-28 CrossRef Medline

22. van Duijn S, Bulk M, van Duinen SG, et al. Cortical iron reflects severity of Alzheimer's disease. J Alzheimers Dis 2017;60:1533-45 CrossRef Medline

23. Bulk M, Abelmoula WM, Nabuurs RJA, et al. Post-mortem MRI and histology demonstrate differential iron accumulation and cortical myelin organization in early and late onset Alzheimer's disease. Neurobiol Aging 2018;62:231-42 CrossRef Medline

24. Birkl C, Carassiti D, Hussain F, et al. Assessment of ferritin content in multiple sclerosis brains using temperature-induced $\mathbf{R}_{2}^{\star}$ changes. Magn Reson Med 2018;79:1609-15 CrossRef Medline

25. Konijn AM, Carmel N, Levy R, et al. Ferritin synthesis in inflammation, II: mechanism of increased ferritin synthesis. Br J Haematol 1981;49:361-70 CrossRef Medline

26. Khoury MN, Alsop DC, Agnihotri SP, et al. Hyperintense cortical signal on magnetic resonance imaging reflects focal leukocortical encephalitis and seizure risk in progressive multifocal leukoencephalopathy. Ann Neurol 2014;75:659-69 CrossRef Medline

27. Brew BJ, Davies NWS, Cinque P, et al. Progressive multifocal leukoencephalopathy and other forms of JC virus disease. Nat Rev Neurol 2010;6:667-79 CrossRef Medline

28. Wattjes MP, Wijburg MT, Vennegoor A, et al; Dutch-Belgian Natalizumab-associated PML study group. MRI characteristics of early PML-IRIS after natalizumab treatment in patients with MS. J Neurol Neurosurg Psychiatry 2016;87:879-84 CrossRef Medline

29. Wattjes MP, Wijburg MT, van Eijk J, et al; Dutch-Belgian Natalizumab-associated PML study group. Inflammatory natalizumab-associated PML: baseline characteristics, lesion evolution and relation with PML-IRIS. J Neurol Neurosurg Psychiatry 2018;89:535-41 CrossRef Medline 